\title{
The Envelope of IRC+10216 Seen at High Angular Resolution
}

\author{
Nicolas Mauron \\ Groupe d'Astrophysique, CNRS and Université de Montpellier, Place \\ Bataillon, 34095 Montpellier, France
}

Patrick J. Huggins

Physics Department, New York University, 4 Washington Place, New York NY 10003, USA

\begin{abstract}
.
Deep optical images of the circumstellar envelope of IRC +10216 have been obtained with CFHT and HST, with angular resolutions of $1^{\prime \prime}$ and $0.1^{\prime \prime}$, respectively. In the outer regions, the envelope is seen in dustscattered galactic light, and is detected up to $\sim 200^{\prime \prime}$ from the star, corresponding to 8000 years of expansion. The envelope is composed of discrete, nearly circular, thin, limb-brightened shells. These shells are spaced at irregular intervals, and the shell/intershell density contrast is typically 3 . Although the HST observations show a distinct bipolar geometry very near the star, the global shell pattern suggests a roughly isotropic, episodic mass loss mechanism.
\end{abstract}

\section{Introduction}

IRC +10216 is the archetype of evolved asymptotic giant stars. Because it is located at $\sim 120 \mathrm{pc}$ from the Sun, it is an exceptional target for high angular resolution studies of stellar and circumstellar phenomena. Its carbon-rich dusty, molecular envelope is the site of numerous physical and chemical processes, such as grain formation, dust and gas dynamical interactions, possibly shocks, gasphase chemistry, photochemistry induced by ambient galactic ultraviolet light, etc. (e.g. Glassgold 1996, Olofsson 1999). An important aspect to investigate is the density structure of this envelope, and especially the departure from an $r^{-2}$ law, where $r$ is the distance to the star, since this smooth behaviour is very often assumed in models.

\section{Observations and results}

Deep optical images were obtained with the Canada-France-Hawaii Telescope, providing a view of the envelope with unprecedented detail. This nebula reflects the external light of the Galaxy and is detected in visible light over $\sim 200 \times 200^{\prime \prime}$ (Fig.1, left panel). This extension corresponds to about 8000 years of expansion. 

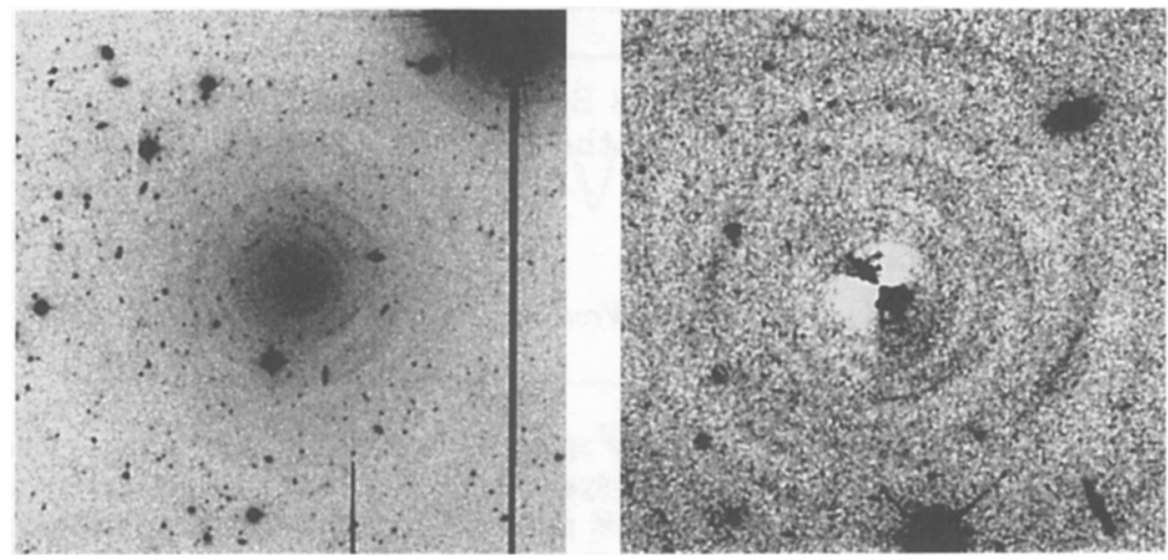

Figure 1. The multiple shells in the circumstellar envelope of IRC+10216. Left panel: CFHT $V$-band image. Field is $223^{\prime \prime} \times 223^{\prime \prime}$, with North to the top, East to the left. Right panel: HST WFPC2 wide$V$ image, with a smooth, radial profile subtracted to enhance visibility of the shells. The field is $74^{\prime \prime} .5 \times 74^{\prime \prime} .5$, rotated $26^{\circ}$ counter-clockwise with respect to left panel.

The observations reveal that the envelope is roughly spherically symmetric, but is very inhomogeneous, with thin nested shells which are circular but incomplete. This outer structure is confirmed by the HST images at $0.1^{\prime \prime}$ resolution (fig.1, right). These shells are seen due to grain scattering, and they are in fair correlation with less clear shell structures seen in the gas in the molecular lines of $\mathrm{CN}$ and $\mathrm{C}_{3} \mathrm{H}$. The shells are more widely separated farther from the star, and their widths increase with radius. Their separation corresponds to $\sim 40$ yrs near the star, and 200-800 yrs far from it. The shell/intershell density contrast is typically 3 and can reach a factor of 10 . Finally, the HST image shows a very clear bipolar structure at the center.

Our results rule out the common assumption of a smooth $r^{-2}$ density law, and point to strong temporal variations of stellar mass loss with a broad range of time scales. A physical explanation for both the central bipolarity and the surrounding roughly isotropic shells is still lacking. More details can be found in the papers by Mauron \& Huggins $(1999,2000)$.

\section{References}

Glassgold, A.E. 1996, Ann. Rev. Ast. Astrophys., 34, 241

Mauron, N., \& Huggins, P.J. 1999, A\&A, 349, 203

Mauron, N., \& Huggins, P.J. 2000, A\&A, 359, 707

Olofsson, H. 1999, in IAU Symposium 191, Asymptotic Giant Branch Stars, ed.

T. Le Bertre, A. Lèbre \& C. Waelkens (IAU-ASP) 